\title{
Prevalência de lesões musculoesqueléticas em lutadores de jiu-jítsu: uma revisão
}

\section{sistemática}

\author{
Prevalence of musculoskeletal injuries in jiu-jitsu fighters: a systematic review \\ Prevalencia de lesiones musculoesqueléticas en luchadores de jiu-jitsu: una revisión sistemática
}

\section{Resumo}

Objetivo: Verificar na literatura científica a prevalência e fatores relacionados às lesões musculoesqueléticas em praticantes de Jiu-Jítsu e descrever quais os segmentos anatômicos mais acometidos. Metodologia: Trata-se de uma revisão sistemática, que tomou como referência o protocolo Preferred Reporting Items for Systematic Reviews and Meta-Analysis (PRISMA), nos Portais de Periódicos da BVS (Biblioteca Virtual em Saúde) e CAPES (Coordenação de Aperfeiçoamento de Pessoal de Nível Superior). Foram incluídos estudos epidemiológicos observacionais que avaliaram a prevalência de lesões ortopédicas em atletas praticantes de Jiu-Jítsu, no período de 2009 a 2020. Resultados: Foram selecionados 4 artigos dos 174 localizados inicialmente, os quais apresentaram como resultados o joelho sendo o segmento anatômico mais acometido. Além disso, o sexo masculino, os atletas iniciantes de faixa branca e azul, e aqueles com idade entre 20 e 30 anos foram os mais acometidos. No entanto, com relação ao tempo de prática, não foi observada prevalência de nenhum período. Conclusão: Nessa revisão sistemática foi observado que há uma alta prevalência da ocorrência de lesões musculoesqueléticas em lutadores de Jiu-Jítsu. O segmento anatômico mais acometido é o joelho e os fatores relacionados variam de acordo com certas variáveis, sendo mais comuns em atletas do sexo masculino, iniciantes e adultos jovens.

Palavras-chave: Lesões; Traumatismo; Artes marciais; Jiu-Jítsu.

\begin{abstract}
Objective: To verify in the scientific literature the prevalence and factors related to musculoskeletal injuries in Jiu-Jitsu practitioners and describe which anatomical segments are most affected. Methodology: This is a systematic review, which took as reference the Preferred Reporting Items for Systematic Reviews and Meta-Analysis (PRISMA) protocol, in the Periodical Portals of the VHL (Virtual Health Library) and CAPES (Personnel Improvement Coordination). Higher Level). Observational epidemiological studies were included that evaluated the prevalence of orthopedic injuries
\end{abstract}


in athletes practicing Jiu-Jitsu, from 2009 to 2020. Results: Four articles were selected from the 174 initially located, which presented as results the knee being the most anatomical segment stricken. In addition, males, beginner white and blue belt athletes, and those aged between 20 and 30 years were the most affected. However, regarding the time of practice, no prevalence of any period was observed. Conclusion: In this systematic review, it was observed that there is a high prevalence of musculoskeletal injuries in Jiu-Jitsu fighters. The most affected anatomical segment is the knee and the related factors vary according to certain variables, being more common in male athletes, beginners and young adults.

Keywords: Injuries; Trauma; Martial Arts; Jiu-Jitsu.

\section{Resumen}

Objetivo: Verificar en la literatura científica la prevalencia y los factores relacionados con las lesiones musculoesqueléticas en practicantes de Jiu-Jitsu y describir cuáles son los segmentos anatómicos más afectados. Metodología: Se trata de una revisión sistemática, que tomó como referencia el protocolo Preferred Reporting Items for Systematic Reviews and Meta-Analysis (PRISMA), en los Portales Periódicos de la BVS (Biblioteca Virtual en Salud) y CAPES (Coordinación de Perfeccionamiento del Personal). Se incluyeron estudios epidemiológicos observacionales que evaluaron la prevalencia de lesiones ortopédicas en deportistas practicantes de Jiu-Jitsu, de 2009 a 2020. Resultados: Se seleccionaron cuatro artículos de los 174 inicialmente localizados, los cuales presentaron como resultados siendo la rodilla el segmento anatómico más afectado. Además, los más afectados fueron el sexo masculino, los atletas principiantes de cinturón blanco y azul y los que tenían entre 20 y 30 años. Sin embargo, en cuanto al tiempo de práctica, no se observó prevalencia de ningún período. Conclusión: En esta revisión sistemática se observó que existe una alta prevalencia de lesiones musculoesqueléticas en los luchadores de Jiu-Jitsu. El segmento anatómico más afectado es la rodilla y los factores relacionados varían según determinadas variables, siendo más frecuente en atletas masculinos, principiantes y adultos jóvenes.

Palabras clave: Lesiones; Trauma; Artes Marciales; Jiu-Jitsu.

\section{Introdução}

A prática do jiu-jítsu tem origem incerta e acredita-se que esse data de 1880 no Japão com o dr. Jigoro Kano (Tegner, 2003). No Brasil, ele foi trazido pelo conde japonês Maeda Koma, para o Pará e acolhido pelo brasileiro Gastão Gracie que deu origem ao Brazilian ou Gracie jiu-jítsu, através do seu desenvolvimento com ênfase nas técnicas de luta no solo, agarramento, imobilização e chaves com movimentos em alavanca, sem o uso de golpes diretos com mãos, pés ou outras partes do corpo (Gouvea et al., 2020; Gurgel, 2007).

Atualmente, segundo a Confederação Brasileira de Jiu-Jítsu (CBJJ), esse esporte é praticado no mundo todo com 2260 academia cadastradas, conduzidas por professores que também são atletas faixas preta registrados na Confederação Brasileira de Jiu-Jítsu (CBJJ) e International Brazilian Jiu-Jitsu Federation (IBJJF) (“CBJJ | Confederação Brasileira de Jiu-Jitsu,” n.d.), tanto como luta para defesa pessoal, como profissão ou mesmo como uma atividade física para manter a saúde. Segundo pesquisa realizada pelo Ministério da Saúde em 2018, a procura por lutas e artes marciais no Brasil aumentou 109\% desde 2006, na qual, segundo o Instituto Brasileiro de Geografia e Estatística (IBGE), cerca de 1,3\% dos brasileiros praticam jiu-jítsu, o que significa algo torno de 2,5 milhões de praticantes (Maciel, 2018).

Segundo Silva Júnior et al., (2018) em estudo realizado com 108 atletas, existe uma alta prevalência de lesões em lutadores de jiu-jítsu, sendo as regiões mais acometidas as articulações do joelho e ombro. Tal cenário se repete em todo o mundo, já que o jiu-jítsu é um esporte de combate e, portanto, pode facilitar a aparecimento de quadros álgicos e a ocorrência de lesões musculoesqueléticas como fraturas, luxações, entroses e contusões, sendo observado um maior acometimento das articulações, decorrente da aplicação de golpes chamados de alavancas articulares ou chaves de articulação (Del Vecchio et al., 2016; Juliano Eustaquio et al., 2021).

Pelo fato de ser uma luta de contato direto, e que, portanto, pode favorecer a ocorrência de lesões musculoesqueléticas que podem interferir significativamente na qualidade vida dos atletas acometidos (B. V. C. Silva, Júnior, Lopes, \& Mota, 2012) há, então, a necessidade de ampliar as investigações quanto as lesões musculoesqueléticas mais frequentes entre lutadores de jiujítsu, visto que elas ocorrem com frequência, e não existem muitos estudos que abordam o assunto. Esta revisão sistemática, portanto, tem por objetivo verificar a prevalência de lesões musculoesqueléticas em praticantes de jiu-jítsu e descrever quais os 
segmentos anatômicos mais acometidos.

\section{Metodologia}

\subsection{Desenho do estudo}

Trata-se de uma revisão sistemática que foi realizada seguindo a metodologia Preferred Reporting Items for Systematic Reviews and Meta-Analysis (PRISMA) (Shamseer et al., 2015).

\subsection{Estratégia de busca}

A pergunta que guiou esta revisão foi formulada seguindo a estratégia PICO; P: praticantes de Jiu-Jítsu, I; prática de jiu-jítsu, C; não intervenção e O: prevalência de lesões musculoesqueléticas.

A busca foi realizada, por assunto, com termos/descritores indexados no Mesh (Medical Subject Headings) e Descritores em Ciências da Saúde (DeCS), "injuries", "Jiu-Jitsu" e "traumatismos em atletas" nos Portais de Periódicos da BVS (Biblioteca Virtual em Saúde) e CAPES (Coordenação de Aperfeiçoamento de Pessoal de Nível Superior), durante os meses de maio e junho de 2020 .

\subsection{Critérios de inclusão e exclusão}

Foram incluídos nesta pesquisa artigos provenientes de estudos observacionais tipo transversais, completos e disponíveis online, nos idiomas português e inglês, que abordaram o tema lesões musculoesqueléticas em lutadores de jiu-jítsu, em que os dados foram coletados a partir da aplicação de questionários ou da realização de entrevistas, e que foram publicados no período de 2009 a 2020. Foram excluídos os estudos na modalidade de revisão, reflexões e, após a análise dos artigos, aqueles que apresentavam resultados inconsistentes, e que não atingiram o percentual estabelecido através da aplicação da ferramenta que avaliou a qualidade deles.

\subsection{Seleção dos estudos}

Os estudos encontrados como resultado da busca foram selecionados individualmente, durante os meses de maio e junho de 2020, através da análise do título e do resumo, de forma que permanecessem aqueles que atendem, direta ou indiretamente a pergunta dessa revisão.

\subsection{Análise de qualidade dos estudos}

Os estudos selecionados foram lidos na integra e analisados a partir de escala STROBE (Malta, Cardoso, Bastos, Magnanini, \& Silva, 2010), a qual é composta por 22 itens divididos em subcategorias pertencentes aos grupos: título e resumo, introdução, métodos e resultados, os quais devem estar presentes em um estudo observacional. A partir dessa, os artigos foram classificados de acordo com sua pontuação, e foram selecionados aqueles que preencheram $80 \%$ dos itens estabelecidos na escala mencionada.

\section{Resultados}

Foram encontrados 174 artigos, e após a leitura dos resumos foram excluídos 131 desses por não tratarem de lesões musculoesqueléticas, abordando lesões em outros sistemas do organismo. Foram excluídos, também, os artigos duplicados, os que não estavam relacionados ao tema proposto, de forma que esses focavam apenas no tratamento das lesões, ou em uma lesão específica, os de caráter de revisão e os textos indisponíveis online. Por fim, foram incluídos apenas os artigos que preencheram mais de $80 \%$ os critérios do STROBE. 
Figura 1 - Fluxograma do processo de seleção do estudo.
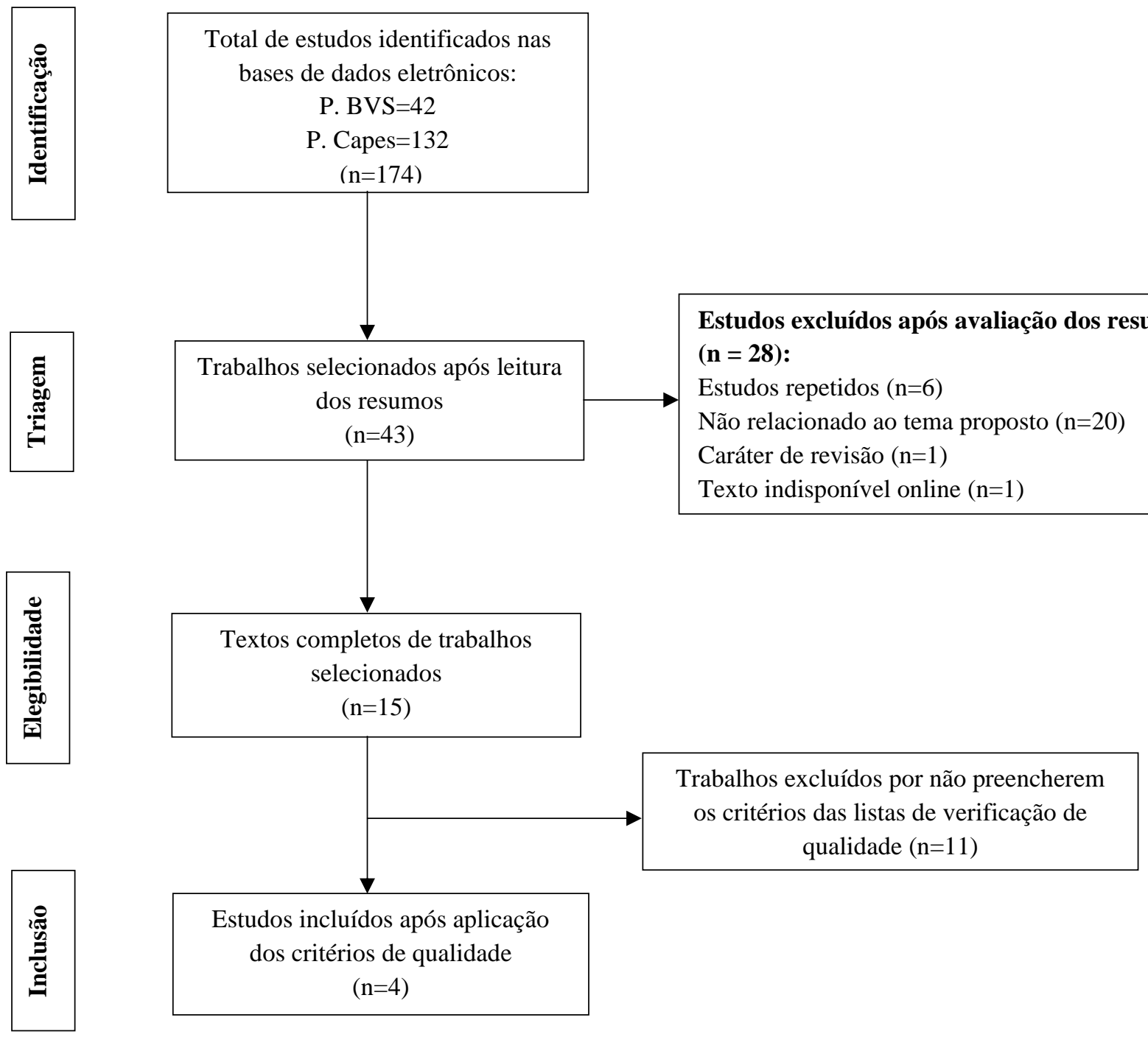

Fonte: Autores.

De forma a caracterizar os artigos que foram analisados, o Quadro 1 traz as informações necessárias para contextualizálos nessa revisão sistemática. 
Quadro 1 - Caracterização dos artigos que compuseram a amostra deste estudo, 2014 - 2019.

\begin{tabular}{|c|c|c|c|c|}
\hline Título do artigo & Autor & $\begin{array}{c}\text { Ano de } \\
\text { publicação }\end{array}$ & País de origem & $\begin{array}{l}\text { Pontuação no } \\
\text { STROBE (\%) }\end{array}$ \\
\hline $\begin{array}{l}\text { Incidence of injury among male brazilian } \\
\text { jiujitsu fighters at the world jiu-jitsu no-gi } \\
\text { championship } 2009\end{array}$ & Kreiswirth et al. & 2014 & EUA & 81 \\
\hline $\begin{array}{l}\text { Prevalência de lesões e avaliação } \\
\text { funcional do movimento entre praticantes } \\
\text { de jiu jitsu }\end{array}$ & Lopes et al. & 2018 & Brasil & 81 \\
\hline Brazilian jiu-jitsu injury in training survey & Petrisor et al. & 2019 & Canadá & 81 \\
\hline $\begin{array}{l}\text { Could current factors be associated with } \\
\text { retrospective sports injuries in brazilian } \\
\text { jiu-jitsu? A cross-sectional study }\end{array}$ & das Graças et al. & 2017 & Brasil & 81 \\
\hline
\end{tabular}

Fonte: Autores.

O Quadro 2 apresenta aspectos relacionados à metodologia de cada artigo. Todos os artigos têm como objetivo em comum descrever a ocorrência de lesões musculoesqueléticas em lutadores de Jiu-Jítsu, porém, cada um em seu contexto particular. Da mesma forma, todos eles se configuram em estudos transversais, e a técnica de obtenção de dados varia entre questionários e entrevistas.

Quadro 2 - Metodologia dos artigos que compuseram a amostra deste estudo, 2014 - 2019.

\begin{tabular}{|c|c|c|c|c|}
\hline Autor & Objetivos & Amostra & $\begin{array}{c}\text { Delineamento } \\
\text { do Estudo }\end{array}$ & Técnica de Coleta de Dados \\
\hline Kreiswirth et al. & $\begin{array}{c}\text { Determinar a incidência de } \\
\text { lesões e risco para ocorrência } \\
\text { dessas em cada nível de faixa e } \\
\text { seguimentos anatômicos em um } \\
\text { tornei internacional de Brazilian } \\
\text { jiujitsu }\end{array}$ & 951 atletas & $\begin{array}{l}\text { Descritivo } \\
\text { transversal }\end{array}$ & $\begin{array}{l}\text { Aplicação de questionário (fatores } \\
\text { analisados: sexo, idade, peso, nível de } \\
\text { faixa, anos de experiência, número de } \\
\text { dias de treinamento por semana e } \\
\text { números de luta no torneio) }\end{array}$ \\
\hline Lopes et al. & $\begin{array}{l}\text { Estudar possíveis relações entre } \\
\text { à prevalência de lesões e o } \\
\text { sistema Funcional do } \\
\text { Movimento em lutadores de Jiu } \\
\text { Jitsu. }\end{array}$ & $\begin{array}{c}31 \\
\text { indivíduos }\end{array}$ & $\begin{array}{l}\text { Analítico } \\
\text { transversal }\end{array}$ & $\begin{array}{l}\text { Aplicação de um questionário com } 04 \\
\text { perguntas fechadas, composto de questões } \\
\text { objetivas com a finalidade de identificar: } \\
\text { 1- A frequência semanal de treino do } \\
\text { participante, } 2 \text { - Se já havia se lesionado, } 3 \\
\text {-Em caso positivo, qual articulação havia } \\
\text { lesionado, } 4 \text { - Há quanto tempo teria } \\
\text { ocorrido a lesão }\end{array}$ \\
\hline Petrisor et al. & $\begin{array}{l}\text { Descrever a ocorrência de } \\
\text { lesões durante os treinos, e } \\
\text { classificar o tipo da lesão e } \\
\text { explorar as características dos } \\
\text { participantes e das lesões } \\
\text { associadas ao desejo de sair do } \\
\text { jiu-jitsu após a lesão. }\end{array}$ & 70 atletas & $\begin{array}{l}\text { Descritivo } \\
\text { transversal }\end{array}$ & $\begin{array}{l}\text { Aplicação } \\
\text { questionário, incluindo perguntas sobre } \\
\text { dados demográficos, lesões em } \\
\text { competição e / ou treinamento, tratamento } \\
\text { recebido e se o participante considerou } \\
\text { descontinuar a JJ após lesão. }\end{array}$ \\
\hline
\end{tabular}




\begin{tabular}{|c|c|c|c|c|}
\hline das Graças et al. & $\begin{array}{l}\text { Analisar alguns elementos } \\
\text { internos, como flexibilidade, } \\
\text { exposição ao treinamento e } \\
\text { medidas antropométricas, bem } \\
\text { como descrever a ocorrência e } \\
\text { as características de lesões } \\
\text { musculoesqueléticas } \\
\text { retrospectivas em diferentes } \\
\text { faixas etárias dos praticantes de } \\
\text { Brazilian Jiu-Jitsu }\end{array}$ & $\begin{array}{c}193 \\
\text { praticantes } \\
\text { de jiu-jitsu }\end{array}$ & $\begin{array}{c}\text { Estudo } \\
\text { transversal }\end{array}$ & $\begin{array}{l}\text { Os participantes foram entrevistados e } \\
\text { avaliados apenas uma vez para obter } \\
\text { informações sobre histórico de prática, } \\
\text { nível de atividade física e ocorrência de } \\
\text { lesão musculoesquelética. Em adição a } \\
\text { isso o Questionário Internacional de } \\
\text { Atividade Física - Formulário Curto } \\
\text { (IPAQ-SF) foi utilizado para determinar a } \\
\text { atividade física total de cada participante. } \\
\text { Posteriormente, foram realizados testes } \\
\text { clínicos para analisar a flexibilidade } \\
\text { articular global e segmentar, a amplitude } \\
\text { de movimento da coluna lombar e a força } \\
\text { de preensão manual. }\end{array}$ \\
\hline
\end{tabular}

Fonte: Autores.

A distribuição da ocorrência de lesões musculoesquelética de acordo com a seguimento anatômico acometido é apresentado na Tabela 1.

Tabela 1 - Seguimentos Anatômicos como diagnóstico de lesões, 2014 - 2019.

\begin{tabular}{lcccc}
\hline $\begin{array}{c}\text { Seguimentos } \\
\text { Anatômicos } \\
\text { Acometidos (\%) }\end{array}$ & Kreiswirth et al. & Lopes et al. & Petrisor et al. & das Graças et al . \\
\hline Ombro & 16,1 & 29 & 49,2 & 19,4 \\
Cotovelo & 19,4 & 26 & 38,5 & 12,5 \\
Joelho & 19,4 & 71 & 50,8 & - \\
Tornozelo & 12,9 & 19 & 36,9 & - \\
Cabeça e pescoço & - & - & 64,3 & 2,4 \\
Costelas & - & 3 & 40 & 12,6 \\
Dedos & - & 9 & 78,5 & - \\
\hline
\end{tabular}

Fonte: Autores.

Segundo Kreiswirth et al., (2014) dentro da amostra de 951 atletas que estavam participando de um torneio, 62 sofreram lesões naquele momento, as quais o artigo define como uma situação em que o lutador solicitou tratamento médico ou não pôde continuar o torneio devido a dor ou desconforto. Nesse contexto, o cotevelo e o ombro foram os seguimentos mais acometidos, cada um representando 19,4\% das lesões. Os autores não relatam possiveis acometimentos das costelas, dedos e, cabeça e pescoço. No artigo de (Lopes et al., 2018), os autores trazem que 100\% dos atletas entrevistados relataram já ter tido pelo menos uma lesão decorrente da prática de jiu-jitsu, e, portanto, os resultados aqui apresentados são baseados na amostra de 31 atletas. Os seguimentos mais acometidos foram o ombro com $29 \%$ e o joelho com $71 \%$ das lesões, e nesse artigo, os autores não relatam acometimento da cabeça e pescoço.

Para Petrisor et al. (2019), os participantes puderam selecionar mais de uma lesão ao realizar o questionário e, portanto, as porcentagens não somam $100 \%$. Nesse contexto, eles trazem que 64,3\% da população que respondeu o questionário já havia 
sofrido uma lesão na cabeça e pescoço e 78,5\% tinha sofrido leões nos dedos, sendo os 2 seguimentos mais acometidos nesse estudo. das Graças et al. (2017), trazem que o ombro foi o seguimento mais acometido, representando 19,4\% das vezes dentro da amostra de todas as lesões analisadas $(\mathrm{n}=247)$. Joelho, tornozelo e dedos não foram analisados individualmente.

Nos resultados do estudo de Kreiswirth et al. (2014), a idade média de atletas lesionados foi de 29,4. Nesse não há relato de intervalos de idade analisados de forma individual. Para Lopes et al. (2018), em que os atletas foram divididos em intervalos de 5 em 5 anos, o que teve a maior quantidade de atletas lesionados foi o de 33 a 38 anos, sendo a idade média de todos eles 30,9 anos. Já para Petrisor et al. (2019), essa divisão não foi em intervalos homogêneos, sendo aqueles com idade entre 18 a 25 anos os mais acometidos por lesão (Tabela 2).

Na pesquisa de das Graças et al. (2017), há uma divisão por idade mais peculiar (Tabela 2). Todos os participantes estão classificados em 3 grupos pertencentes a faixas etárias diferentes: os "adolescentes", aqueles que tem de 12 a 15 anos, os “adultos" entre 22 e 27 anos e os "masters" que são os que estão entre 32,8 e 38 anos. Nesse contexto, o intervalo mais acometido foi o dos "adultos" com ocorrência de 108 lesões.

Tabela 2 - Caracterização da idade dos participantes por artigo selecionado 2014 - 2019.

\begin{tabular}{|c|c|c|c|c|}
\hline & Kreiswirth et al. & Lopes et al. & Petrisor et al. & das Graças et al. \\
\hline Média de idade (anos) & 29,4 & 30,9 & NR & NR \\
\hline $\begin{array}{l}\text { Intervalos de idade } \\
\text { analisados }\end{array}$ & NR & $\begin{array}{l}18-23 \\
23-28 \\
28-33 \\
33-38 \\
38-43\end{array}$ & $\begin{array}{c}18-25 \\
25-29 \\
30-34 \\
35-39 \\
40-44 \\
45-49 \\
50-54 \\
>55\end{array}$ & $\begin{array}{c}12-15 \\
22-27 \\
32,8-38\end{array}$ \\
\hline $\begin{array}{l}\text { Intervalo com maior } \\
\text { frequência de lesões }\end{array}$ & NR & $33-38$ & $18-25$ & $22-38$ \\
\hline
\end{tabular}

Fonte: Autores.

Kreiswirth et al. (2014), não apresentam a divisão por sexo dos participantes do seu estudo. Lopes et al. (2018), não incluíram em seu estudo o sexo feminino, justificando ter sido para evitar vieses decorrente das diferenças anatômicas, e, portanto, sua amostra foi $100 \%$ do sexo masculino (Tabela 3 ).

Tabela 3 - Frequência de lesões musculoesqueléticas estratificadas de acordo com o sexo dos participantes, 2014 - 2019.

\begin{tabular}{cccc}
\hline & Lopes et $\boldsymbol{a l}$. & Petrisor et al. & das Graças et al. \\
\hline Feminino & - & 7 & 35 \\
Masculino & 31 & 63 & 158 \\
Total (n) & 31 & 70 & 193 \\
\hline
\end{tabular}

Fonte: Autores. 
Kreiswirth et al. (2014) não incluíram atletas de faixa branca no estudo. As faixas que tiveram mais atletas acometidos foram as faixas azul e preta. Lopes et al. (2018) não incluíram nenhum atleta iniciante (faixas brancas e azul) no estudo, e relataram predominância da faixa preta com 48 atletas lesionados (Tabela 4).

Petrisor et al. (2019) foi o único estudo analisado que avaliou a distribuição de lesões em todos os níveis de faixa. Dessas, as mais acometidas foram as iniciantes, sendo a azul com 30 atletas lesionados, seguida pela branca com 26 . No estudo, das Graças et al. (2017) fazem uma divisão diferenciada no que se trata a distribuição de atletas lesados em níveis de faixa. Eles apresentam o número de atletas com lesão apenas no maior e menor níveis de faixa (preta e branca, respectivamente) e reúne todos ou outros níveis em um só grupo (Tabela 4).

Tabela 4 - Nível de faixa dos atletas que sofreram lesão por artigo selecionado, 2014 - 2019.

\begin{tabular}{ccccc}
\hline $\begin{array}{c}\text { Graduação } \\
\text { (faixa) }\end{array}$ & Kreiswirth et al. & Lopes et al. & Petrisor et al. & das Graças et al. \\
\hline Branca & - & - & 26 & 106 \\
Azul & 21 & - & 30 & 77 \\
Roxa & 11 & 36 & 7 & 3 \\
Marrom & 12 & 16 & 4 & 10 \\
Preta & 18 & 48 & & \\
\hline
\end{tabular}

Fonte: Autores.

Com relação ao tempo de prática do Jiu-Jitsu, apenas (Petrisor et al., 2019) trouxeram tal informação conforme apresentado na Tabela 5, na qual não houve nenhum intervalo de tempo que mostrou uma predominância de atletas lesionados.

Tabela 5 - Tempo de Prática dos Participantes - 2019.

\begin{tabular}{ccc}
\hline & Tempo de prática em anos & Número de atletas \\
\hline & $0-1$ & 13 \\
Petrisor et al. & $1-3$ & 16 \\
& $3-5$ & 12 \\
& $5-7$ & 13 \\
\hline
\end{tabular}

Fonte: Autores.

\section{Discussão}

Este estudo analisou as evidências científicas acerca da prevalência de lesões musculoesqueléticas em praticantes de jiu-jitsu e os segmentos anatômicos mais acometidos. A arte marcial em questão é baseada em seis técnicas: projeções, imobilizações, chaves, torções e estrangulamento, todas fundadas em princípios biomecânicos e podem causar traumas severos aos praticantes do esporte (Scoggin et al., 2014; J. E. Silva, Voltoline et al., 2012). Provavelmente, devido a própria técnica da luta que expõe os praticantes a quedas e choques com grande aplicação de força, a incidência de lesões ortopédicas acaba por se tornar muito comum na prática do esporte onde, aproximadamente, $90 \%$ dos atletas participantes relataram a ocorrência de, pelo menos, uma lesão (Dumont et al., 2018; B. V. C. Silva et al., 2012).

Na presente revisão sistemática, foi encontrada uma maior prevalência no acometimento do joelho quando comparada a outros seguimentos anatômicos, corroborando com os achados de outros estudos (Enohi et al., 2015; Kreiswirth et al., 2014; 
McDonald et al., 2017). No entanto, quando analisada a ocorrência dessas lesões de acordo com variáveis demográficas, não foi possível identificar um fator comum que influenciou significativamente a ocorrência de lesões musculoesqueléticas em lutadores de Jiu-Jítsu. Essa situação também se aplica a outras variáveis, como o tipo de lesão mais prevalente, analisadas em outros artigos que não puderam ser incluídos nessa revisão, na qual não há uma concordância quanto qual seria a mais frequente (Carvalho et al., 2013; Stephenson et al., 2018).

Com relação aos locais das lesões, como já mencionado, todos os estudos selecionados identificaram o joelho como o sendo a região mais acometida, provavelmente, pela técnica da luta que é baseada em quedas, imobilizações e bloqueios articulares (Nicolini et al., 2021), na qual a maior parte da luta ocorre no chão, em que o atleta prefere se manter apoiado nos joelhos para manter a guarda (Del Vecchio et al., 2016), e que, apesar de não expor o lutador a golpes diretos, como socos ou chutes, o expõe a lesões de articulações, principalmente o joelho.

Quando observado a idade dos atletas que tiverem mais lesões, os estudos analisados não apresentam um padrão quanto a sua abordagem, mas permitem ter uma estimativa da média de idade mais acometida, na qual observa-se uma maior prevalência de lesões em adultos jovens, em torno de 20 a 30 anos. Segundo (Moriarty et al., 2019), a faixa de idade mais acometida por lesões variou entre 26 a 35 anos, enquanto para (J. E. Silva et al., 2012) a média de idade foi de 29,6 anos. Assim, ambos os resultados corroboram para com os achados dessa revisão.

Em relação a variável sexo, os estudos selecionados demonstraram uma prevalência maior do sexo masculino. Esse achado parece ser devido ao fato de que há mais pessoas do sexo masculino praticando o esporte, no geral, provavelmente por uma questão cultural. Poucos são os estudos que realizam a análise dividida por sexo, devido à escassez de atletas do sexo feminino presentes em academias, escolas e competições, no entanto, aqueles que analisaram uma população composta por homens e mulheres contém um número consideravelmente maior de atletas do sexo masculino (Del Vecchio et al., 2016; Moriarty et al., 2019; Oliveira et al., 2010).

No que diz respeito à faixa, que caracteriza o nível dos atletas, os estudos apontam para um maior acometimento das faixas iniciantes (branca e azul). Esse episódio se repete no estudo realizado por (Silva Júnior et al., 2018), considerando a época de treinamento, e na pesquisa realizada por (Oliveira et al., 2010). Esses achados podem ser devido ao fato de que os atletas iniciantes não dominam as técnicas da luta necessárias para realizar golpes como raspagens, imobilizações e até quedas de maneira segura e eficaz.

Por fim, referente ao tempo de prática do esporte, apenas um dos estudos que compuseram a amostra abordou sobre esta variável. E nesse, não foi possível verificar uma maior prevalência de nenhum intervalo de tempo especificamente, podendo, esse fator, ser influenciado pelo nível do atleta, a forma como ele pratica o esporte, se de forma casual ou com finalidade de competir, ou mesmo em que academia e com que professor ele pratica. De maneira similar, segundo Moriarty et al. (2019), não há um padrão estabelecido entre a ocorrência de lesões e o tempo que o atleta pratica o esporte, sendo o intervalo de 1 a 4 anos o que apresentou mais lutadores com lesões nesse estudo.

A presente revisão sistemática apresentou limitações devido à escassez de artigos que atendessem a avaliação dos critérios de qualidade do STROBE e que abordassem outras variáveis como o tipo de lesão mais prevalente, os mecanismos ou golpes em que ocorreram as lesões, comparação com o nível do adversário ou o peso do atleta. Todas essas variáveis possuem, também, o potencial de influenciar de maneira significativa a ocorrência de lesões musculoesqueléticas em lutadores de Jiu-Jítsu. Além disso, não houve consideração de fatores étnicos raciais e socioeconômicos dos participantes, diminuindo a capacidade de generalização a partir dos resultados dos estudos que compuseram a amostra desta investigação.

Necessita-se, então, de estudos observacionais mais detalhados quanto ao perfil socioeconômico dos lutadores pertencentes à amostra para que se obtenha uma análise mais precisa acerca dos fatores que possam favorecer a ocorrência de lesões ortopédicas nessa população, visto que a literatura traz evidências, apenas, referentes a fatores físicos e técnicos. 


\section{Conclusão}

Nessa revisão sistemática foi observado que há uma alta prevalência da ocorrência de lesões musculoesqueléticas em lutadores de Jiu-Jítsu. O segmento anatômico mais acometido é o joelho e os fatores relacionados variam de acordo com certas variáveis, sendo mais comuns em atletas do sexo masculino, iniciantes e adultos jovens com idade variando entre os 20 e 30 anos. Futuros trabalhos de investigação que levem em consideração uma análise temporal da incidência e prevalência das lesões musculoesqueléticas, com desenhos de estudos adequados, são necessárias, sobretudo, para que os modelos de aula e treinamento sejam ajustados, resultando em menor exposição a lesões pelos seus praticantes.

\section{Referências}

Carvalho, J. P., Grecco, L. H., \& Oliveira, A. R. (2013). Prevalência de lesões em praticantes de jiu-jítsu: comparação entre nível iniciante e avançado. Science in Health, 4(9), 71-79.

CBJJ | Confederação Brasileira de Jiu-Jitsu. (n.d.). https://cbjj.com.br/

das Graças, D., Nakamura, L., Barbosa, F. S. S., Martinez, P. F., Reis, F. A., \& Oliveira-Junior, S. A. (2017). Could current factors be associated with retrospective sports injuries in Brazilian jiu-jitsu? A cross-sectional study. BMC Sports Sci Med Rehabil, 9(1), 1-10.

Del Vecchio, F. B., Gondim, D. F., \& Arruda, A. C. P. (2016). Functional Movement Screening Performance of Brazilian Jiu-Jitsu Athletes from Brazil: Differences Considering Practice Time and Combat Style. J Strength Cond Res, 30(8), 2341-7.

Dumont, R. P., Matos, R. S., Nunes Filho, J. C. C., Pinto, D. V, Caminha, J. S. R., \& Nunes, M. P. O. (2018). Incidência de lesões em praticantes recreacionais de jiu-jitsu. Coleção Pesquisa Em Educação Física, 17(3), 71-78.

Enohi, R. T., \& Lucena, R. R. (2015). Prevalência de lesões musculoesqueléticas em praticantes de jiu jitsu. Rev Cientifica Integrada, 2(2), 1-15.

Gouvea, C. V. de A., Miguel, G. C. de, Neto, M. E., \& Rodrigues, G. U. (2020). Lesões ortopédicas decorrentes da prática de jiu-jitsu: síntese de evidências clínicas. International Journal of Health Management Review, 6(2), 1-12. https://ijhmreview.org/ijhmreview/article/view/222

Gurgel, F. (2007). Brazilian Jiu-Jitsu: Manual Pessoal de Jiu-Jítsu Do Iniciante ao Avançado (1st ed., Vol. 2). São Paulo: Gurgel F.

Juliano Eustaquio, J. M., Fontoura Borges, A. M., Vilela, L. S., Carvalho Gouveia, M. P., Rabelo, A. L., Kaleka, C. C., ... Barbosa Neto, O. (2021). Does the Fight Profile Interfere with Orthopedic Injuries in Brazilian Jiu-Jitsu? Open Access Journal of Sports Medicine, Volume 12, $171-178$.

Kreiswirth, E. M., Myer, G. D., \& Rauh, M. J. (2014). Incidence of injury among male brazilian jiujitsu fighters at the world jiu-jitsu No-Gi championship 2009. J Athl Train, 49(1), 89-94.

Lopes, O. P. S., Alves, L. D. B., Felipe, P. N. F., Neto, P. S. P., \& Souza, S. F. M. (2018). Prevalência de lesões e avaliação funcional do movimento entre praticantes de Jiu Jitsu. Motricidade, 14(1), 368-75.

Maciel, V. (2018). Corrida e artes marciais crescem entre os brasileiros — Português (Brasil). https://www.gov.br/saude/ptbr/assuntos/noticias/2018/dezembro/corrida-e-artes-marciais-crescem-entre-os-brasileiros

Malta, M., Cardoso, L. O., Bastos, F. I., Magnanini, M. M. F., \& Silva, C. M. F. P. (2010). STROBE initiative: guidelines on reporting observational studies. Rev Saude Publica, 44(3), 559-65.

McDonald, A. R., Murdock, F. A., McDonald, J. A., \& Wolf, C. J. (2017). Prevalence of Injuries during Brazilian Jiu-Jitsu Training. Sports, 12 (5), 39.

Moriarty, C., Charnoff, J., \& Felix, E. R. (2019). Injury rate and pattern among Brazilian jiu-jitsu practitioners: A survey study. Phys Ther Sport, 39, 107-13.

Nicolini, A. P., Penna, N. A., De Oliveira, G. T., \& Cohen, M. (2021). Epidemiology of orthopedic injuries in jiu-jitsu athletes . Acta Ortopédica Brasileira, 29(1), 49-53.

Oliveira, E. G., Oliveira, R. R. C., \& Silva, P. K. A. F. (2010). Prevalência e incidência de lesões em atletas participantes do campeonato open de jiu-jitsu da cidade de catalão-go. Rev Eletrônica Saúde CESUC, 1(1).

Petrisor, B. A., Del Fabbro, G., Madden, K., Khan, M., Joslin, J., \& Bhandari, M. (2019). Brazilian Jiu-Jitsu Injury in Training Survey. Sports Health, 11(5), $432-439$.

Scoggin, J. F., Brusovanik, G., Izuka, B. H., Van Rilland, E. Z., Geling, O., \& Tokumura, S. (2014). Assessment of injuries during brazilian jiu-jitsu competition. Orthop J Sport Med, 2(2), 1-7.

Shamseer, L., Moher, D., Clarke, M., Ghersi, D., Liberati, A., Petticrew, M., \& Shekelle, P. S. L. (2015). Preferred reporting items for systematic review and meta-analysis protocols (PRISMA-P) 2015: elaboration and explanation. BMJ, 349, g7647.

Silva, B. V. C., Júnior, M. M., Lopes, C. R., \& Mota, R. M. (2012). Brazilian Jiu-Jitsu: Aspectos do Desempenho. Rev Bras Prescrição e Fisiol Do Exerc, 6(31), $195-200$.

Silva, J. E., Voltoline, J. C., \& Mine, C. E. C. (2012). Fatores associados às lesões de atletas do brazilian jiu-jitsu no vale do paraíba paulista. Rev Bras Prescrição e Fisiol Do Exerc, 10(60), 25-31. 
Research, Society and Development, v. 11, n. 3, e17111326345, 2022

(CC BY 4.0) | ISSN 2525-3409 | DOI: http://dx.doi.org/10.33448/rsd-v11i3.26345

Silva Júnior, J. N., Kons, R. L., Dellagrana, R. A., \& Detanico, D. (2018). Prevalência de lesões em atletas de Brazilian jiu-jitsu: comparação entre diferentes níveis competitivos. Brazilian J Kinanthropometry Hum Perform, 20(3), 280-9.

Stephenson, C., \& Rossheim, M. E. (2018). Brazilian Jiu Jitsu, Judo, and Mixed Martial Arts Injuries Presenting to United States Emergency Departments, 20082015. J Prim Prev, 39(5), 421-435.

Tegner, B. (2003). Guia Completo de Jiu-Jitsu 483 Fotos de Golpes, Instruções Passo a Passo (1st ed.). Rio de Janeiro: Record. 